\title{
The Resource Allocation Algorithm of LTE Systems Based on Proportional Fair Particle Swarm
}

\author{
Yunxiao $\mathrm{Zu}^{1,2}$, Jing Gao, Jingbo Guo ${ }^{2}$, Lin Shao \\ ${ }^{1)}$ School of Electronic Engineering, Beijing University of Posts and Telecommunications, Beijing 100876, China \\ (zuyx@ @ bupt.edu.cn) \\ ${ }^{2)}$ State Key Lab. of Power System, Tsinghua University, Beijing 100084, China (guojb@mail.tsinghua.edu.cn)
}

\begin{abstract}
Resource scheduling technology is an important part of radio resource management. The resource allocation for real-time traffic in LTE systems is studied in this paper. The system model is established based on margin adaptive criterion. The proportional fair criterion is added to the particle swarm algorithm, and for the real-time traffic, the particle swarm optimization algorithm of resource allocation in LTE systems is developed based on proportional fair. The simulation results show that the particle swarm optimization algorithm based on proportional fair is correct and valid.
\end{abstract}

Keywords — LTE, Resource allocation, proportional fair, particle swarm

\section{基于比例公平粒子群的 LTE 系统资源分配算法 ${ }^{*}$}

\author{
㸖云霄 ${ }^{1,2}$ 高婧 郭静波 $^{2}$ 邵林 \\ 1) 北京邮电大学电子工程学院, 北京 100876 \\ 2) 清华大学电力系统国家重点实验室，北京 100084
}

摘 要 资源调度技术是无线资源管理的重要部分, 本文对 LTE 系统中实时业务的资源分配问题进行了研究。建立了基于余量自适 应准则的系统模型, 将比例公平准则引入到粒子群算法中, 提出了面向实时业务、基于比例公平的 LTE 系统资源分配的粒子群优化算 法，通过仿真验证了所提算法的正确性和优越性。

关键词 LTE，资源分配，比例公平，粒子群

\section{1. 引言}

LTE 是面向 $4 \mathrm{G}$ 发展起来的一种通信网络, 具有用户数 量多、信道环境复杂、业务种类多、用户移动范围广等一 系列特点。因此, 对网络资源进行合理分配, 保证用户的 服务质量 (Quality of Service, QoS) 成为研究的重点内容 之一。LTE 系统的核心技术是 OFDM, 因此就涉及子载波 的合理分配和使用。相关领域的学者对 LTE 系统的资源分 配进行了部分研究, Jang and Lee 提出了通过将子载波优先 分配给具有最好信道增益的用户, 从而使系统容量最大化 的优化算法 ${ }^{[1]}$, 但是, 该算法的缺点是如果用户间的信道增 益差别很大, 则较高信道增益的用户将占用几乎所有的信 道, 从而使得其他用户不能进行数据的传输。文献[2]研究 了最大-最小问题用以保证所有用户具有相同的数据速率,
但是, 却没有考虑实际应用中不同用户可能需要不同的传 输速率。文献[3]提出了一种次优算法, 首先假设用户进行 等功率分配, 然后根据用户的传输速率比将子载波首先分 配给最合适的用户，但是这用算法的计算复杂度较大。

本文将粒子群算法应用于 LTE 系统的子载波和功率分 配算法的研究中, 针对 LTE 系统中的实时性业务进行研究, 提出了一种基于比例公平粒子群的资源分配算法, 使得在 总功率和误码率一定的情况下, 通过给每个子载波上分配 一定数量的比特, 最大化系统吞吐量。

\section{2. 基于粒子群算法的问题描述}

粒子群优化算法 (Particle Swarm Optimization, PSO) 最初起源于对鸟群受食行为的模拟, 即鸟群如何在一片未

* 本项工作得到北京市科委项目（Z111100066511007）和清华大学电力系统国家重点实验室项目（SKLD11KM05）的资助。 
知的区域中找到食物, 并找到一条最近的路线。目前, 该 算法已经广泛应用到各个领域中, 比如多目标优化、模式 识别、数据的聚类和分类、约束优化、生物系统建模、多 峰值函数优化等问题 ${ }^{[4]}$ 。粒子群优化算法将要解决的问题比 喻为待求解的粒子, 每个粒子都有一个速度, 粒子依照此 速度在区域中移动, 每个优化问题的解都被比喻为一只寻 找食物的鸟。

粒子群优化算法的本质是在可能的解空间中寻找最优 可行解。设解空间为 $P$ 维, 则最终可行解的大小与 $P$ 中元 素的大小有关。由于每个元素的取值不同, 并且每个元素 的取值对最终解都有影响, 因此, 将每一个可能解看作一 个 $N$ 维向量, 所有可能解的集合构成解空间。在实数空间 $R^{n}$ 中, 系统性能优化问题的可行解表示为空间中移动的粒 子的位置。设第 $i$ 个粒子在上一时刻所处的位置为 $x_{i}(t-1)$, 当前时刻所处的位置为 $x_{i}(t)$, 速度为 $v_{i}(t)$, 则位置关系式为

$$
x_{i}(t)=x_{i}(t-1)+v_{i}(t)
$$

由于鸟是有记忆的, 所以, 这里的粒子也是有记忆的。 第一步, 每个粒子都会将自己每次飞过的最好的点 pbest $_{i}$ 记录下来, 并将路径信息分享给其余粒子; 第二步, 通过 比较所有的 $p b e s t_{i}$, 得到所有 $p b e s t_{i}$ 的最小值, 即最好的 位置, 这就是全局最优解, 记为 $g b e s t$ 。每个粒子每次的 飞行速率和方向都会同时受到 pbest ${ }_{i}$ 和 $g$ best 的影响, 并 将不断地靠近这两个点, 速度的计算公式为

$$
\begin{aligned}
v_{i}(t)= & w \times v_{i}(t-1)+c_{1} \times \text { rand }_{1} \times \text { pbest }_{i}-x_{i}(t-1) \\
& +c_{2} \times \text { rand }_{2} \times\left[\text { gbest }^{-x_{i}}(t-1)\right]
\end{aligned}
$$

其中, $w$ 为惯性因子, $c_{1}$ 和 $c_{2}$ 为加速度因子, 分别用 于调节局部信息的权值和全局信息的权值, rand 和 $\operatorname{rand}_{2}$ 为两个随机数, 用于增加种群的多样性, 且 $0 \leq$ rand $_{1}$, rand $_{2} \leq 1$ 。位置和速度的变化范围分别为 $\left[-x_{\text {max }} x_{\text {ma a }}\right]$ 和 $\left[-v_{\text {max }}, v_{\text {max }}\right]$, 这两个限制范围很重要,

因为粒子速度过大, 会有可能飞过最优解位置, 过小又可 能导致局部最优, 所以, 通常设 $v_{\text {max }}=x_{\text {max }}$, 作为速度的 最大约束范围。

在 LTE 的资源分配中, 主要涉及子载波、比特、系统 吞吐量、功率等。针对研究的资源分配算法, 对应到粒子 群算法中, 鸟, 亦即 “粒子” 就是 “所有子载波”, “食物” 就是系统的 “总吞吐量”, “鸟” 飞行的 “路线” 就是 “每 个子载波” 的比特数。优化目标就是找到一种在满足约束
条件下, 使所有子载波上的比特数之和最大, 也就是系统 容量最大的子载波分配算法。

\section{3. 基于余量自适应优化准则的系统模型}

资源分配和功率分配属于调度问题, 调度算法根据每 个子信道的信道质量, 选择合理的调制编码方式, 满足一 定的误码率和系统总发射功率等要求。其实质就是限定条 件下的优化问题。主要的优化准则有以下两种 ${ }^{[5]}$ : (1) 速率 自适应 (Rate Adaptive, RA) 优化准则一一总发射功率和 误比特率一定的条件下, 最大化系统吞吐量; (2) 余量自 适应 (Margin Adaptive, MA) 准则——数据传输速率和误 比特率一定的条件下, 最小化系统的总发射功率。

由于实时类业务对传输速率、误码率有明确的要求, 因此采用余量自适应准则优化准则 (Margin Adaptive, MA) 对实时类业务建模。

假设 LTE 系统中有 $K$ 个用户, 系统带宽被划分为 $N$ 个 子载波, 在信道估计良好的前提下, 进行子载波分配。令 $h_{k, n}$ 表示第 $k$ 个用户在第 $n$ 个子载波上的瞬时信道增益, $\rho_{k, n}$ 表示第 $n$ 个子载波被第 $k$ 个用户占用的情况, $\rho_{k, n}=1$ 表示被占用, $\rho_{k, n}=0$ 表示没有被占用, $b_{k, n}$ 表示第 $k$ 个 用户占用第 $n$ 个子信道时被分配的比特数, $R_{k}$ 表示第 $k$ 个 用户的速率, 则根据 MA 优化准则的系统数学模型如下, 其中式 (3) 表示目标函数, 式 (4) 表示约束条件。

$$
\min P_{T}=\sum_{k=1}^{K} \sum_{n=1}^{N} \rho_{k, n} \cdot h_{k, n}
$$

$$
\begin{aligned}
& \mathrm{C} 1: \sum_{k=1}^{K} \rho_{k, n} \leq 1, \quad \rho_{k, n} \in\{0,1\}, \quad \forall k, n \\
& \mathrm{C} 2: p_{k, n}=N_{0} / 3 \cdot\left[Q^{-1}\left(P_{\mathrm{e}} / 4\right)\right]^{2}\left(2^{C_{k, n}}-1\right), \quad \forall k, n(4) \\
& \mathrm{C} 3: \sum_{n=1}^{N} \rho_{k, n} b_{k, n} \geq R_{k}, \quad \forall k
\end{aligned}
$$

式 (4) 中, C1 表明多个用户不能共享同一个子载波, $\mathrm{C} 2$ 表明了系统功率和子载波分配比特的关系表达式, C3 表明了各个用户速率的约束规则。

\section{4. 基于比例公平的粒子群算法}

资源分配和功率分配属于调度问题, 调度算法根据每 个子信道的信道质量, 选择合理的调制编码方式, 满足一 定的误码率和系统总发射功率等要求。其实质就是限定条 件下的优化问题。主要的优化准则有以下两种 ${ }^{[5]}$ : (1) 速率 自适应 (Rate Adaptive, RA) 优化准则一一总发射功率和 
误比特率一定的条件下, 最大化系统吞吐量; (2) 余量自 适应 (Margin Adaptive, MA) 准则一一数据传输速率和误 比特率一定的条件下, 最小化系统的总发射功率。

\section{1 比例公平准则}

随着研究的深入, 一些公平性指标也被引入到优化准 则中, 于是出现了第三种优化准则- - 比例公平

(Proportional Fair, PF) 优化准则。比例公平准则综合考 虑了当前的信道条件以及用户过去的平均数据速率, 更贴 近真实世界的需要, 但实现也更复杂 ${ }^{[6-9]}$ 。比例公平准则考 虑为每一个等待服务的用户分配一个调度优先级 $p_{i}(t)$, $p_{i}(t)$ 值越大优先级越高 ${ }^{[10]}, p_{i}(t)$ 定义如下:

$$
p_{i}(t)=\frac{C_{i, n}(t)}{R_{i}(t)}, \quad i=1,2, \cdots, N
$$

其中, $C_{i, n}(t)$ 表示用户 $i$ 在子载波 $n$ 上加载的比特数; $R_{i}(t)$ 表示用户 $i$ 在时刻 $t$ 之前的平均数据速率。

$$
\begin{aligned}
& c_{i, n}(t)=\log _{2}\left\{1+\frac{3 P_{i, n} \cdot g_{i, n}}{N_{0}\left[Q^{-1}\left(\frac{B E R_{i}}{4}\right)\right]^{2}}\right\} \\
& R_{i}(t)=\left(1-\frac{1}{\tau_{i}}\right) R_{i}(t-1)+\frac{1}{\tau_{i}} C_{i, n}(t-1)
\end{aligned}
$$

其中, $P_{i, n}$ 是用户 $i$ 在子载波 $n$ 上的发送功率; $g_{i, n}$ 是用户 $i$ 在该时刻的信道增益; $N_{0}$ 是噪声功率; $B E R_{i}$ 是用户 $i$ 的目标误比特率; $\tau_{i}$ 为用户 $i$ 的平均速率更新因子; $Q^{-1}(\cdot)$ 是 $Q(\cdot)$ 的反函数。

$$
Q(x)=\frac{1}{\sqrt{2 \pi}} \int_{x}^{\infty} e^{-\frac{z^{2}}{2}} d z
$$

由于速率的更新影响信道的质量, 所以, $\tau$ 的取值至关 重要, 必须同时具备两个条件: 满足不同业务对时延的要 求; 能根据当前用户信道条件更新小尺度快速衰落 ${ }^{[10]}$ 。

从公式(5)可知, 在多个用户的蜂窝小区中, 某用户在 某时刻占用频谱、时隙资源时, 用户的小尺度衰落最小, 此时服务该用户，整个系统的吞吐量会有很大的提高; 另 一方面, 当某一用户 $i$ 的信道质量在一段连续的时间内一直 较好时, 那么该用户将一直占用信道资源不释放, 这样造 成的后果就是该用户的平均数据速率 $R_{i}(t)$ 逐渐变大, 根据 公式 (5) 可知, 当 $R_{i}(t)$ 变大时, 该用户的优先级 $p_{i}(t)$ 将
会变小, 这样就会导致该用户的调度终止, 无法再获得服 务。但是, 通过这种机制, 保证了所有用户都有机会被服 务, 信道质量较好的用户占据信道资源时, 系统自动降低 其优先级, 保证信道质量差的用户也可以获得服务机会, 使得用户之间的公平性大大提高。正因为如此, $\mathrm{PF}$ 算法在 移动通信系统中被广泛应用, 也是本文引入比例公平的原 因。

\section{2 基于比例公平的粒子群算法}

传统的粒子群算法如下: 假设搜索次数为 $G$, 初始阶 段, 随机生成初始粒子群大小为 $M$, 向量长度为 $N$, 并且 随机生成 $M$ 个粒子的最佳路径, 并记录 $M$ 个粒子的全局最 佳路径。搜索阶段, 在不超出最大功率范围内, 计算并记 录每个粒子搜索的最佳子载波分配路径, 记录最佳路径, 更新比特分配矩阵, 最终可得到全局最优路径。

将比例公平准则与粒子群算法结合, 在记录每个粒子 的最佳路径后, 先按照比例公平准则进行比例约束, 然后 再记录全局最佳路径。比例公平粒子群算法流程如图 1 所 示。

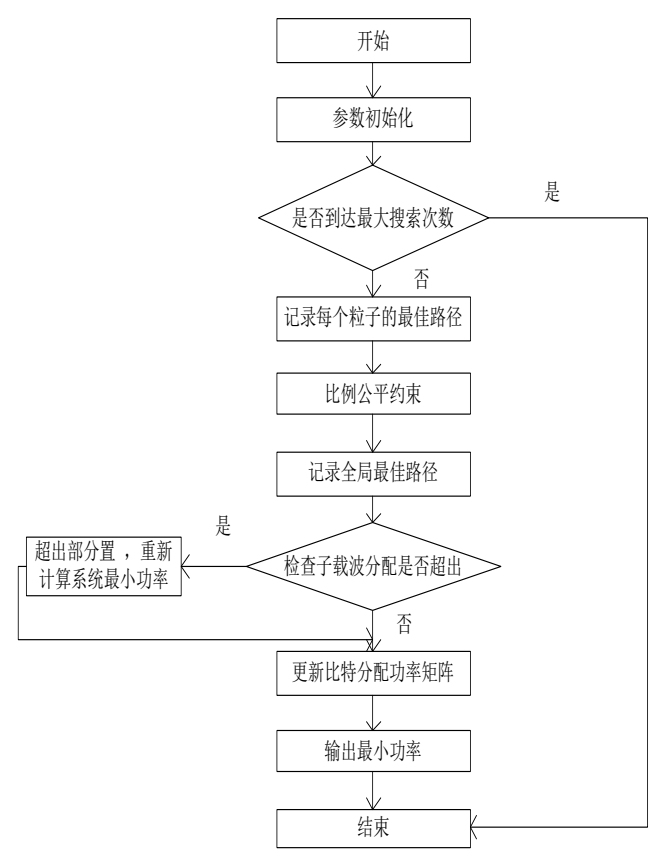

图 1 比例公平粒子群算法流程

\section{5. 仿真结果及分析}

本文对算法的仿真参数选择主要参考 $3 \mathrm{GPP}$ 标准 ${ }^{[11-13]}$ 中的 LTE 参数标准, 具体如下: LTE FDD/TDD 每个子载波 带宽为 $15 \mathrm{kHz}$, 带宽为 $5 \mathrm{MHz}$, 即系统中包含 25 个资源块。 由于 1 个资源块包含 12 个子载波, 所以系统共包含 300 个 子载波。仿真中将 300 个子载波分配给 8 个用户, 选择最 
高调制阶数为 6 , 最大代数为 50 , 系统最大发射功率已知。 在实际系统中, 虽然不同的业务传输速率不同, 但传统的 粒子群算法并未考虑这一点, 本文使用比例公平准则对传 统粒子群算法进行改进, 假设系统用户数据传输速率 $\mathrm{R}=[64,136,144,160,136,96,160,128]$ 。图 2 给出了传统粒子群 算法和实施比例公平的粒子群算法的功率性能。

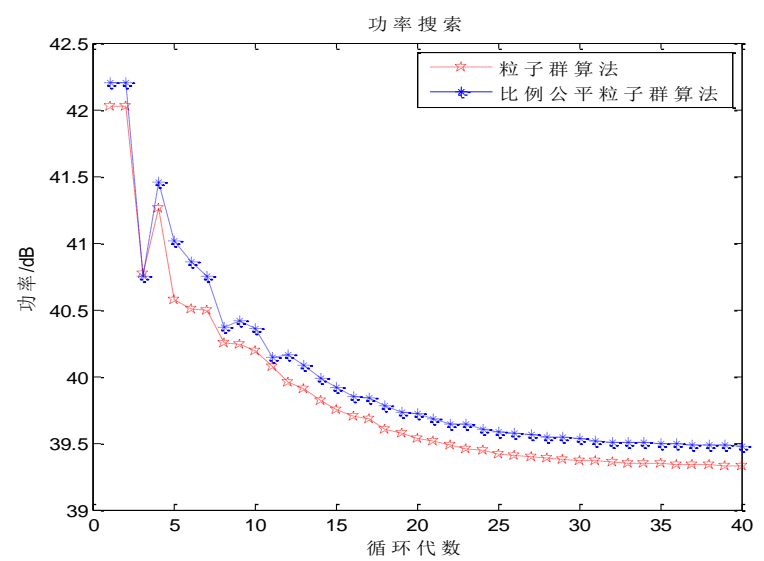

图 2 粒子群算法功率

从图 2 中可以看出, 实施比例公平后, 系统功率得到 提升。根据比例公平准则可知, 载波的比特数越少, 功率 越小。由于传统粒子群算法不考虑实际传输速率, 只是使 功率最小化, 因而功率性能最佳, 但是实际用户对数据传 输速率有要求, 所以实施比例公平后, 系统总功率有所提 升。

图 3 给出了传统粒子群算法和实施了比例公平的粒子 群算法时每个用户的实际传输速率。

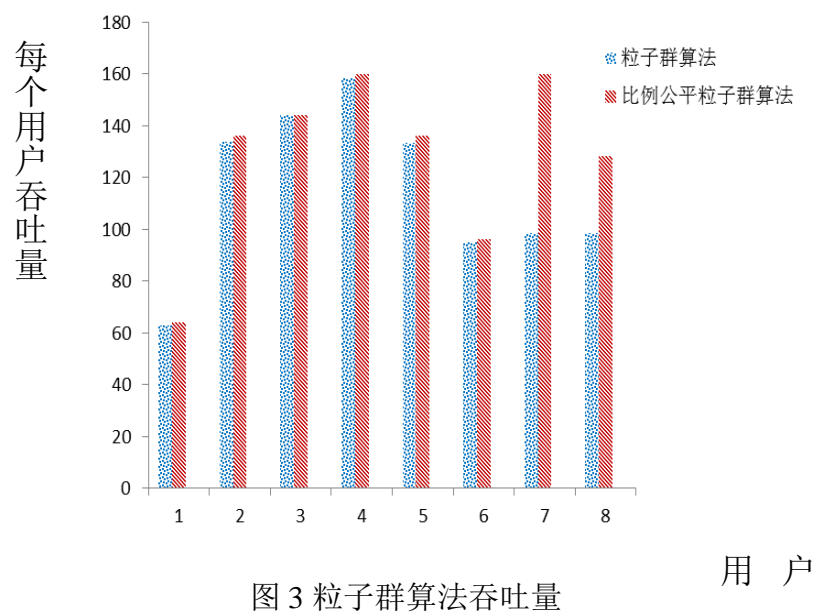

从图 3 中可以看到, 比例公平粒子群算法的实际传输 速率, 也就是吞吐量, 达到了预设的数据传输速率, 即 $\mathrm{R}=[64,136,144,160,136,96,160,128]$, 实现了资源的合理利
用。而传统粒子群算法数据传输速率是随机的, 它只追求 带宽资源的最佳分配, 并不考虑业务的实际需求, 这样可 能导致数据量大的业务得不到很好的服务, 数据量小的业 务占用更多的功率，造成系统资源的浪费。

\section{6. 结论}

本文针对 LTE 系统中的关键技术一一资源调度技术进 行了研究, 根据实时业务的特点, 采用余量自适应准则进 行建模, 并将比例公平准则引入到粒子群算法中, 通过仿 真说明了基于比例公平的粒子群算法相对于传统的粒子群 算法在功率和系统吞吐量方面都得到改善。

\section{参考文献(References)}

[1] J. Jang, K. B. Lee. Transmit power adaptation for multiuser OFDM systems. IEEE J. Sel. Areas Commun. 2003, 21(2): 171-178.

[2] W. Rhee, J. M. Cioffi. Increasing in capacity of multiuser OFDM system using dynamic subchannel allocation. IEEE VTC, 2000, 2(5): 1085-1089.

[3] Zukang Sheng, Jeffrey G. Andrews and Brian L. Evans. Adaptive resource allocation in multiuser OFDM systems with proportional rate constraints. IEEE Tran. Wireless Commun. 2005, 4(12): 2726-2737.

[4] Chen Dong, Multi-objective optimization based on swarm intelligence and game strategy algorithm research. [master thesis]. Hunan University, 2010.

[5] Shen Chenchen. Adaptive resource allocation algorithms OFDMA system. [master thesis]. Dongnan University, 2007.

[6] Fu Junfeng. The Packet Scheduling Algorithm in HSDPA. World Telecommunications, 2006, 4:47-51.

[7] Qian Yu. Research on Key Radio Resource Management Technology In 3G LTE. [master thesis]. Beijing University of Posts and Telecommunications, 2007.

[8] S.Lu, V.Bharghavan, R.Srikant. Fair Scheduling in Wireless Packet Networks. ACM SIGCOMM,1997.8, 188 193

[9] P.Goyal, H.M.Vin, H.Cheng. Start-time Fair QueuingA Scheduling Algorithm for Integrated Services Packet Switching Networks. IEEE/ACM Transactions on Networking, 1997.5, 508 521.

[10] Gu Xiaoping. The Research of Multi-User Scheduling of RRM for LTE. [master thesis]. Nanjing University of Posts and Telecommunications, 2012.

[11] 3GPP 36.321-2008. Packet Data Convergence Protocol (PDCP) Specification[S]. 2008.9.

[12] 3GPP 36.322-2008. Radio Link Control (RLC) Protocol Specification[S]. 2008.9.

[13] 3GPP 36.323-2008. Medium Access Control (MAC) Protocol Specification[S]. 2008.9. 